07

\title{
Модель образования дискретных дислокаций термоупругого мартенситного превращения
}

\author{
(C) Г.А. Малыгин \\ Физико-технический институт им. А.Ф. Иоффре, \\ Санкт-Петербург, Россия \\ E-mail: malygin.ga@mail.ioffe.ru \\ Поступила в Редакцию 12 октября 2020 г. \\ В окончательной редакции 12 октября 2020 г. \\ Принята к публикации 19 октября 2020 г.
}

В рамках теории неравновесных фазовых переходов первого рода Гинзбург-Ландау и ряда разумных предположений впервые рассматривается вопрос перехода предмартенситной наноструктуры кристалла с ЭПФ в структуру дискретных дислокационных петель мартенситного превращения. При решении уравнения $Г-Л$ получены два его асимптотических решения: одно для гомогенного источника наномодуляции кристалла, второе - для пространственно-периодической предмартенситной наноструктуры. Анализ этих решений показал, что консолидация локальных упругих полей наноструктуры может быть причиной образования дискретных дислокационных петель мартенситного превращения.

Ключевые слова: сплавы с ЭПФ, предмартенситная наноструктура, неравновесный фазовый переход, дискретные дислокации мартенситного превращения.

DOI: 10.21883/FTT.2021.02.50477.220

\section{1. Введение}

Сразу после открытия сплавов со сдвиговым механизмом мартенситных переходов и эффектом памяти формы (ЭПФ) [1] была высказана идея, что подобно двойникующим дислокациям фазовый переход аустенит-мартенсит осуществляется движением по межфазной границе специальных дислокаций мартенситного превращения (МП) [2,3]. Но авторами [2,3] не была предложена теоретическая модель образования этих дислокаций. С тех пор прошло 70 лет, но одиночные дислокации МП в их дискретной форме экспериментально до недавних пор никто не наблюдал, из-за их слабой электронной контрастности и высокой скорости перемещения, порядка скорости поперечных волн в кристалле, в отличие от обычных решеточных дислокаций, легко обнаруживаемых трансмиссионной электронной микроскопией. Эксперимент фиксировал лишь существование межфазных границ и наличие на них ступенек атомного размера [4], которые авторы идентифицировали как места пересечения дислокаций МП с поверхностью кристалла. Неизвестными при этом оставались источники дискретных дислокаций МП и динамика их работы. Ситуация изменилась с появлением высокопроизводительных компьютерных устройств и технологий. Они позволили на атомно-молекулярном уровне промоделировать процесс образования дислокаций МП; и с наносекундным разрешением по времени проследить за динамикой их образования.

Таким наиболее продвинутым по результату исследованием является работа [5]. Объектом молекулярнодинамического моделирования служил нанокристаллический сплав TiNi. После приложения нагрузки к сплаву авторы наблюдали зарождение петель дислокаций МП в центре нанозерен, которые, расширяясь по плоскости габитуса, исчезали в границе зерна. При снятии напряжения имела место обратная картина: петли МП зарождались в границе нанозерна и исчезали в его центре. По мере накопления петель в нанозернах формировались межфазные границы. Диаграммы деформации при прямом и обратном мартенситных переходах образовали в макромасштабе гистерезисную петлю. Таким образом, результаты компьютерного моделирования наглядно продемонстрировали на атомном уровне, что дислокации МП являются элементарными носителями обратимой пластической деформации сплавов с ЭПФ.

Компьютерный эксперимент - это эксперимент в условиях, обычно не достижимых в реальном физическом эксперименте. Как и реальный, виртуальный эксперимент, расширяя наши знания, не отвечает на вопрос, почему и как происходит формирование этих петель. Ответ на него должна дать теория, в рассматриваемом случае - теория неравновесных фазовых переходов первого рода Гинзбурга-Ландау $(\Gamma-Л)$. Ее универсальный характер был недавно продемонстрирован в обзоре [6]. В настоящей работе сделана попытка на основе указанной теории разработать модель работы гомогенного источника, генерирующего дискретные дислокационные петли мартенситного превращения. Вначале в разделе 2 приведены основные термодинамические соотношения для равновесных фазовых переходов первого рода. Затем

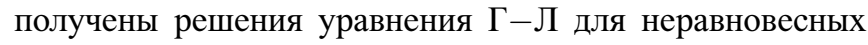
переходов первого рода в двух асимптотических приближениях (раздел 3). Наконец, в разделе 4 обсуждаются условия, при которых предмартенситная наноструктура кристалла эволюционирует в структуру дискретных дислокаций МП. 


\section{2. Термодинамика фазовых переходов первого рода}

Основное термодинамическое соотношение для свободной энергии $\Delta F$, описывающее фазовый переход, как первого, так и второго рода, имеет вид [7]:

$\Delta F=\Delta F_{0}+U_{1} \varphi_{1}+U_{2} \varphi_{2}+U_{12}\left(\varphi_{1}, \varphi_{2}\right)-k_{\mathrm{B}} T \Delta S\left(\varphi_{1}, \varphi_{2}\right)$,

где $\varphi_{1}$ и $\varphi_{2}=1-\varphi_{1}-$ объемные доли, соответственно, предмартенситной фазы и аустенита, $U_{1} \varphi_{1}$ и $U_{2} \varphi_{2}-$ собственные энергии фаз, $U_{12}\left(\varphi_{1}, \varphi_{2}, g\right)=U_{0} \varphi_{1} \varphi_{2}\left(1-g \varphi_{1} \varphi_{2}\right)$ - энергия взаимодействия фаз, $g$ - стрикционный параметр, определяющий возникновение упругих деформаций в решетке, $\Delta F_{S}=k_{\mathrm{B}} T\left(\varphi_{1} \ln \varphi_{1}+\varphi_{2} \ln \varphi_{2}\right)$ - энтропийный вклад в свободную энергию фазового перехода, $T$ - температура, $k_{\mathrm{B}}$ - постоянная Больцмана, $\Delta F_{0}-$ свободная энергия сплава в отсутствие фаз, $\varphi_{1}=\varphi_{2}=0$. Вводя обозначения

$$
\eta=\frac{\varphi_{1}-\varphi_{2}}{\varphi_{1}+\varphi_{2}}, \quad f=\frac{4 \Delta F}{U_{0}}, \quad T_{0}=\frac{U_{0}}{2 k_{\mathrm{B}}},
$$

получаем зависимость свободной энергии в безразмерном виде $f$ от параметра порядка $\eta$, температуры $T$ и стрикционного фактора $g$ [7]

$$
\begin{aligned}
f(\eta, T, g) & =f_{0}+f_{1} \eta-\left(1-\frac{1}{2} g\right) \eta^{2} \\
& +\frac{T}{T_{0}}[(1+\eta) \ln (1+\eta)+(1-\eta) \ln (1-\eta)],
\end{aligned}
$$

где $f_{0}=f(0, T, g), f_{1}=2\left(U_{1}-U_{2}\right) / U_{0}$. Переход системы в новое равновесное состояние определяется условиями

$$
\frac{\partial \Delta f}{\partial \eta}=0, \quad \frac{\partial^{2} \Delta f}{\partial \eta^{2}} \geq 0
$$

где $\Delta f=f-f_{0}$. Применяя эти условия, находим в неявном виде зависимость параметр порядка от температуры и стрикционного фактора $g$,

$$
\begin{gathered}
\frac{T}{T_{c}}=2 \eta\left(1+c \eta^{2}\right)\left(\ln \frac{1+\eta}{1-\eta}\right)^{-1}, \\
T_{c}=\left(1-\frac{1}{2} g\right) T_{0}, \quad c=\frac{g / 2}{1-g / 2} .
\end{gathered}
$$

При $g=0$ из (4a) следует температурная зависимость параметра порядка при фазовом переходе второго рода в рамках модели самосогласованного среднего поля

$$
\frac{T}{T_{c}}=2 \eta\left(\ln \frac{1+\eta}{1-\eta}\right)^{-1}, \quad T_{c}=T_{0} .
$$

Анализ показывает [7], что фазовый переход первого рода возникает при значениях стрикционного параметра $g>0.5$, когда на кривой прямого и обратного переходов возникает гистерезис, и вместо асимметричных ( $\lambda$-образных) производных $d \eta / d T$, появляются симметричные $\Lambda$-образные производные [8].

\section{3. Уравнение Гинзбурга-Ландау и его решение}

При $\eta \ll 1$ энергия (2a) имеет стандартный вид разложения Ландау

$$
f(\eta, T, g)=f_{0}+f_{1} \eta+f_{2} \eta^{2}+f_{4} \eta^{4}+\ldots,
$$

где

$$
\begin{gathered}
f_{2}=\frac{T-T_{c}}{T_{0}}, \quad T_{c}=\left(1-\frac{1}{2} g\right) T_{0}, \\
f_{4}=\frac{T-T_{1}}{6 T_{0}}, \quad T_{1}=\frac{3}{2} g T_{0} .
\end{gathered}
$$

В уравнениях (5) от температуры и стрикционного параметра $g$ зависят только коэффициенты $f_{2}$ и $f_{4}$. На рис. 1 показаны температурные зависимости этих коэффициентов при $g=1.8$. Видно, что почти для всех температур коэффициент $f_{2}>0$, а $f_{4}$ отрицателен в значительном температурном интервале. Принимая во внимание в (5а) только соответствующие слагаемые, получаем уравнение Г-Л [6] для неравновесного фазового перехода в виде нелинейного дифференциального уравнения

$$
\begin{gathered}
\tau \frac{\partial \eta}{\partial t}=\lambda_{d}^{2} \nabla^{2} \eta-\frac{\partial \Delta f(\eta)}{\partial \eta}, \\
\Delta f(\eta)=f_{2}(T, g) \eta^{2}-\left|f_{4}(T, g)\right| \eta^{4},
\end{gathered}
$$

где $t-$ время, $\tau=\lambda_{d} / u_{s}-$ характерное время релаксации параметра порядка, $\lambda_{d}-$ расстояние, определяющее его пространственный градиент, $u_{s}$ - скорость поперечных волн в кристалле. Поскольку гомогенный источник дислокаций МП испускает круговые петли [5], используем полярную систему координат. Лапласиан в

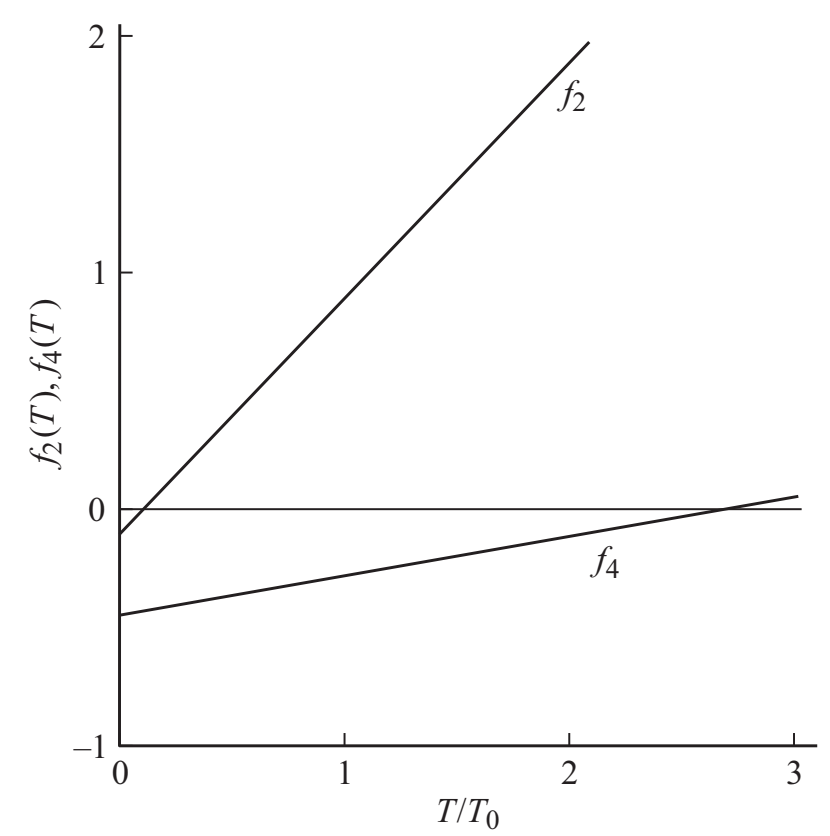

Рис. 1. Зависимость коэффициентов $f_{2}$ и $f_{4}$ от температуры согласно соотношениям (5) при $g=1.8$. 
уравнении (6) имеет в этом случае вид (в компактной и развернутой форме)

$$
\nabla^{2} \eta=\frac{1}{r}\left(\frac{\partial}{\partial r}\left(r \frac{\partial \eta}{\partial r}\right)\right)=\frac{1}{r} \frac{\partial \eta}{\partial r}+\frac{\partial^{2} \eta}{\partial r^{2}} .
$$

Нелинейный характер уравнения (6) с переменной $\eta$, зависящей от координаты и времени, не позволяет найти общее решение этого уравнения (типа уравнения Панлеве) в замкнутом виде. Развернутая форма лапласиана (7) указывает на возможность асимптотического подхода к решению уравнения (6).

3.1. Гомогенный источник наномодуляции (НМ) кристалла. Согласно (7), вблизи источника НМ кристалла $(r=0)$ доминирует компонента $r^{-1}(\partial \eta / \partial r)$. Ее доминирование нивелируется при $r \rightarrow \infty$. Следовательно, в рассматриваемом асимптотическом приближении имеем следующее нелинейное уравнение для $\eta$

$$
\tau \frac{\partial \eta}{\partial t}=\frac{\lambda_{d}^{2}}{r} \frac{\partial \eta}{\partial r}-2 f_{2} \eta+4\left|f_{4}\right| \eta^{3}
$$

Введя нестационарную координату $z_{1}(r, t)=\left(r / \lambda_{d}\right)^{2}-t / \tau$, получаем нелинейное уравнение первого порядка

$$
3 \frac{\partial \eta}{\partial z_{1}}=2 f_{2} \eta-4\left|f_{4}\right| \eta^{3} .
$$

Его решение

$$
\int_{\eta}^{\eta_{0}} \frac{d \eta}{2 f_{2} \eta-4\left|f_{4}\right| \eta^{3}}=\frac{1}{3} z_{1}
$$

позволяет найти зависимость параметра порядка от времени и радиуса источника в виде следующего соотношения

$$
\begin{gathered}
\eta_{1}(r, t)=\left[\frac{2 f_{2} A\left(\eta_{0}\right) \exp \left(-4 f_{2} z_{1}(r, t) / 3\right)}{1+4\left|f_{4}\right| A\left(\eta_{0}\right) \exp \left(-4 f_{2} z_{1}(r, t) / 3\right)}\right]^{1 / 2}, \\
A\left(\eta_{0}\right)=\frac{\eta_{0}^{2}}{2 f_{2}-4\left|f_{4}\right| \eta_{0}^{2}}
\end{gathered}
$$

где $\eta_{0} \neq 0$ - постоянная интегрирования, имеющая смысл критической величины гомогенного источника, когда он начинает функционировать как источник НМ кристалла. На рис. 2 показана зависимость параметра порядка $\eta_{1}(r, t)$ от радиуса $r$ источника при $T / T_{0}=0.5$, $g=1.8, \eta_{0}=0.01$ для трех моментов времени: $t / \tau=0$ (кривая 1$), t / \tau=1$ (кривая 2) и $t / \tau=2$ (кривая 3). Видно, что с увеличением времени источник расширяется. Его расширению способствуют также снижение температуры и величины стрикционного параметра $g$ и увеличение критической величины источника $\eta_{0}$. При этом во всех случаях параметр порядка $\eta_{1}$ экспоненциально стремится к нулю при $|r| \rightarrow \infty$.

3.2. Предмартенситная наноструктура кристалла. Согласно уравнениям (5) и (6) во втором асимптотическом случае уравнение $Г-Л$ для параметра порядка

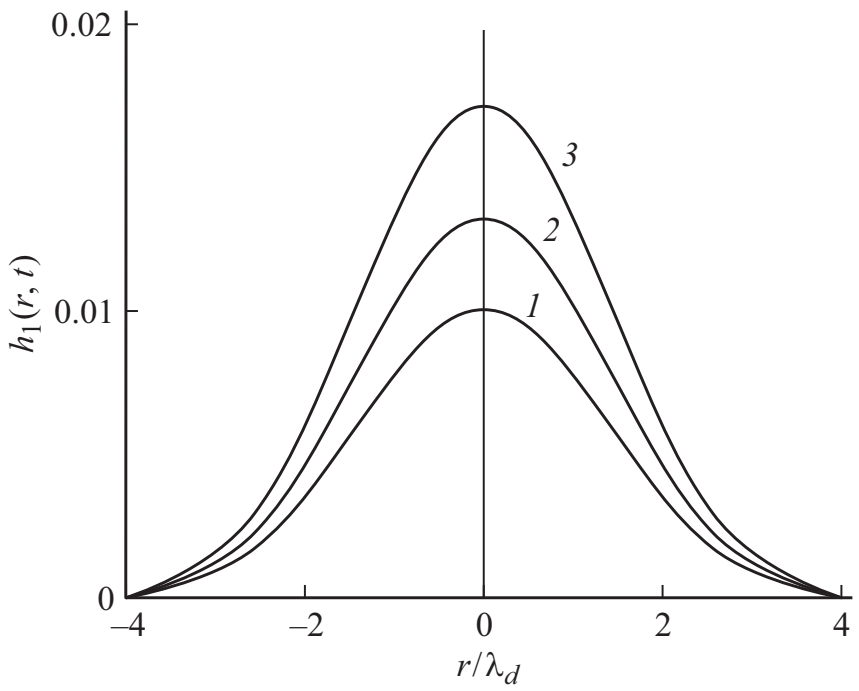

Рис. 2. Вариация ширины гомогенного источника предмартенситной наномодуляции кристалла со временем $t / \tau: 0,1$ и 2 (кривые $1-3$ соответственно) при температуре $T / T_{0}=0.5$, $g=1.8, \eta_{0}=0.01$.

$\eta\left(z_{2}\right)$, где $z_{2}=r / \lambda_{d}-t / \tau$, имеет вид

$$
\frac{\partial^{2} \eta}{\partial z_{2}^{2}}=\frac{\partial \Delta f}{\partial \eta}
$$

Используя тождество $\partial^{2} \eta / \partial z^{2}=2^{-1} \partial(\partial \eta / \partial z)^{2} / \partial \eta$, получаем уравнение для параметра порядка $\eta\left(z_{2}\right)$,

$$
\begin{gathered}
\frac{d \eta}{\sqrt{2} d z_{2}}=\left(f_{2} \eta^{2}-\left|f_{4}\right| \eta^{4}-f_{0}\right)^{1 / 2} \\
=\left|f_{4}\right|^{1 / 2}\left[\left(h_{1}^{2}-\eta^{2}\right)\left(\eta^{2}-h_{2}^{2}\right)\right]^{1 / 2}, \quad(10 \mathrm{~b}) \\
h_{1,2}^{2}=\frac{f_{2}}{\left|2 f_{4}\right|}\left[1 \pm(1-\beta)^{1 / 2}\right], \quad \beta=\frac{4\left|f_{4}\right|}{f_{2}^{2}} f_{0}, \quad \beta<1,
\end{gathered}
$$

где $h_{1,2}$ - корни уравнения $(10 \mathrm{~b}), f_{0}-$ постоянная интегрирования. Решением уравнения (10b) является эллиптический интеграл первого рода

$$
F(\theta, k)=\frac{z_{2}+z_{0}}{\Lambda_{0}},
$$

$\Lambda_{0}=\left[f_{2}\left(1+(1-\beta)^{1 / 2}\right)\right]^{1 / 2} F(\pi / 2, k), \quad k=\left(1-\frac{h_{2}^{2}}{h_{1}^{2}}\right)^{1 / 2}$,

где $k$ - модуль интеграла, $\Lambda_{0}=\Lambda / \lambda_{d}-$ безразмерный период наноструктуры, $z_{0}-$ постоянная интегрирования. В терминах эллиптических функций Якоби $s n(x)$ зависимость параметра порядка от радиуса и времени имеет вид

$$
\eta_{2}(r, t)= \pm h_{1}\left[1-k^{2} s^{2}\left(\frac{\pi}{2}\left(\frac{z_{2}(r, t)+z_{0}}{\Lambda_{0}}\right)\right)\right]^{1 / 2} .
$$




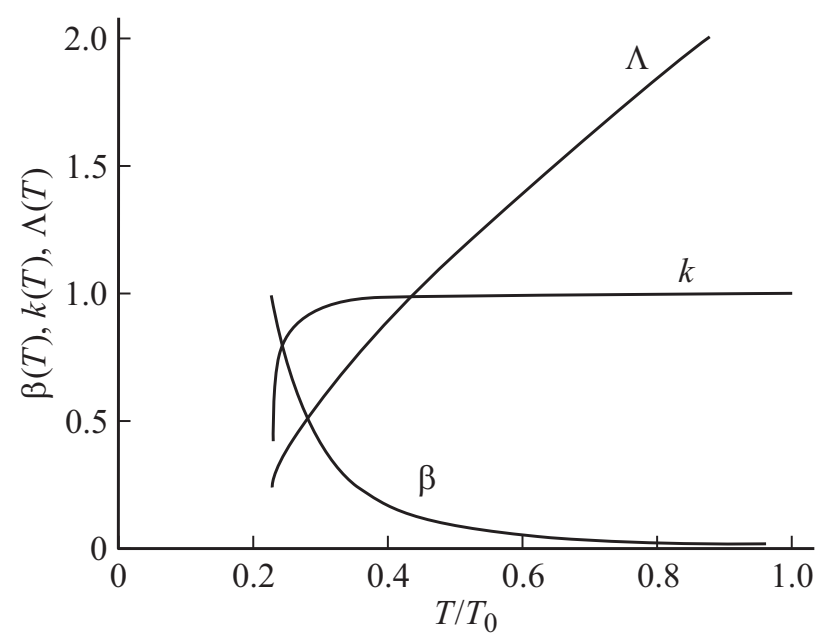

Рис. 3. Температурные зависимости параметров предмартенситной наноструктуры кристалла согласно соотношениям (10) и (11) при $g=1.8$ и $f_{0}=0.01$.
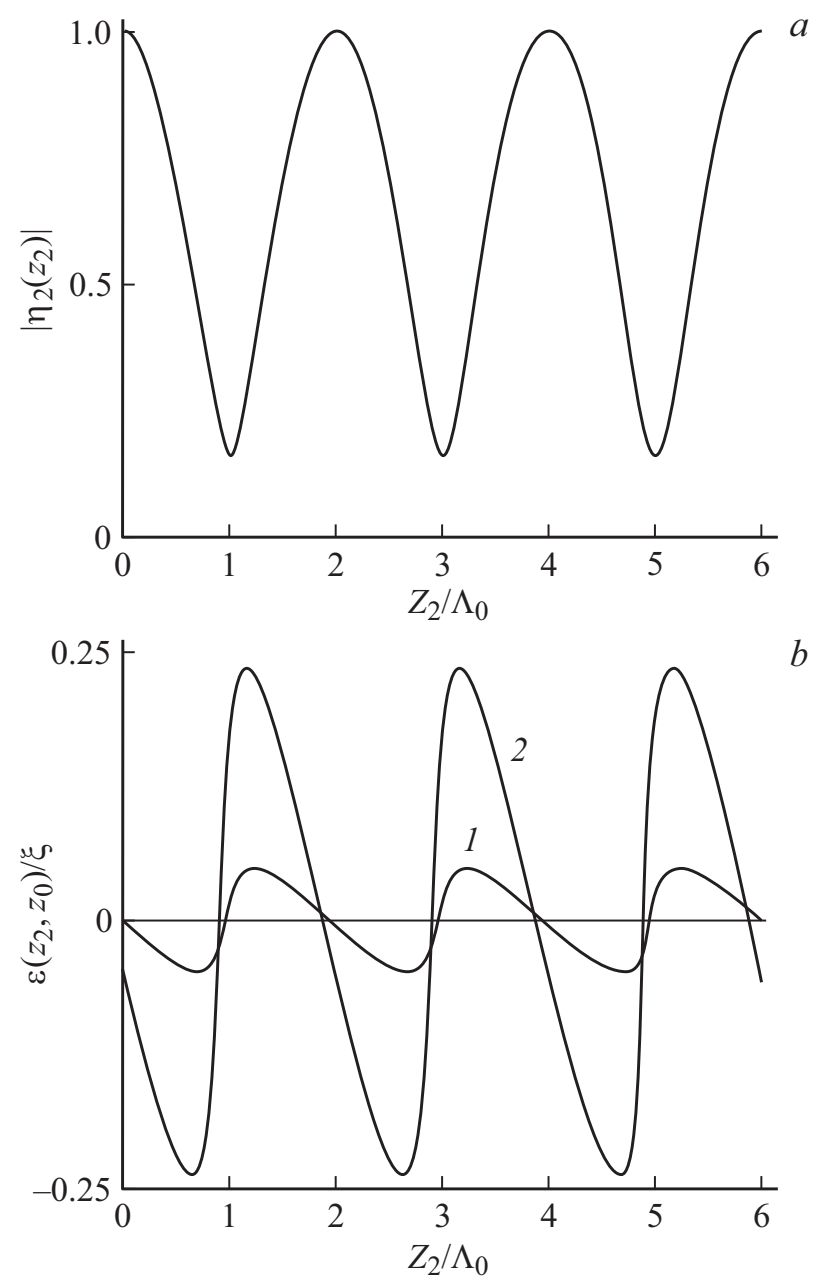

Рис. 4. Пространственно-периодическая наноструктура кристалла согласно уравнению (11b) (a); (b) смешанная из двух альтернативных по знаку уравнений (11b) наноструктура (12) при сдвиге по фазе между ними $z_{0}=0.1$ (кривая 1) и 0.5 (кривая 2).
Температурные зависимости модуля $k$, параметра $\beta<1$ и периода наноструктуры $\Lambda=\lambda_{d} \Lambda_{0}\left(\lambda_{d}=0.4 \mathrm{~nm}\right)$ при $g=1.8$ и $f_{0}=0.01$ показаны на рис. 3. Видно, что при указанных значениях параметров наноструктура образуется при температурах выше критического значения $T / T_{0}=0.23$. Оценка периода наноструктуры $\Lambda$ находится в диапазоне наблюдаемых в эксперименте предмартенситных наноструктур с твидовым (tweed) или апельсиновой корки рельефами поверхности кристалла. Рис. 4, $a$ демонстрирует пространственно-периодическую зависимость параметра порядка $\pm \eta_{2}\left(z_{2}\right)$ согласно уравнению (11b) при $T / T_{0}=0.5, z_{0}=0$ и указанных на рис. 3 значениях параметров $g$ и $f_{0}$. Деформация кристалла, связанная с образованием микс-наноструктуры из альтернативных по знаку $\eta_{2}^{ \pm}$решений (11b) уравнения (9), имеет вид [7]

$$
\varepsilon\left(z_{2}, z_{0}\right)=\xi\left[\eta_{2}^{+}\left(z_{2}+z_{0}\right)+\eta_{2}^{-}\left(z_{2}\right)\right],
$$

где $\xi$ - дисторсия решетки кристалла при неоднородном распределении параметра порядка, $z_{0}-$ сдвиг по фазе между альтернативными по знаку решениями уравнения (9). На рис. $4, b$ показана зависимость $\varepsilon\left(z_{2}\right)$ в координатах $\varepsilon\left(z_{2}, z_{0}\right) / \xi-z_{2} / \Lambda_{0}$ при $z_{0}=0.1$ (кривая 1 ) и 0.5 (кривая 2). Видно, что при указанных значениях $z_{0}$ сдвиг фаз влияет в основном на амплитуду предмартенситных деформаций кристалла.

\section{4. Дислокации мартенситного превращения}

Результаты молекулярно-динамического моделирования наноисточника дислокаций МП в кристалле сплава TiNi [5] наглядно демонстрируют, что элементарный носитель мартенсита - это дислокации мартенситного превращения. Приведенные в разделе 2 термодинамические расчеты базируются на характерных (фундаментальных) решениях нелинейного дифференциального уравнения $Г-Л$. Они указывают, на наш взгляд, путь для теоретического, аналитического решения вопроса об источнике дислокаций МП и механизме их образования. В настоящем разделе рассматривается возможная модель перехода предмартенситной наноструктуры кристалла (11)-(12) в структуру дискретных дислокаций МП. В основе модели лежат три предположения: 1) время релаксации упругих напряжений больше, чем время образования предмартенситных деформаций [9], 2) дискретные дислокации МП результат консолидация определенного числа упругих предмартенситных деформаций (12) и 3) вклад каждого радиального слоя в общую сумму убывает с ростом радиуса $r$, как $1 / r$.

В результате сделанных предположений, получаем следующее уравнение для расчета структуры дискретных дислокаций, генерируемых гомогенным источником 

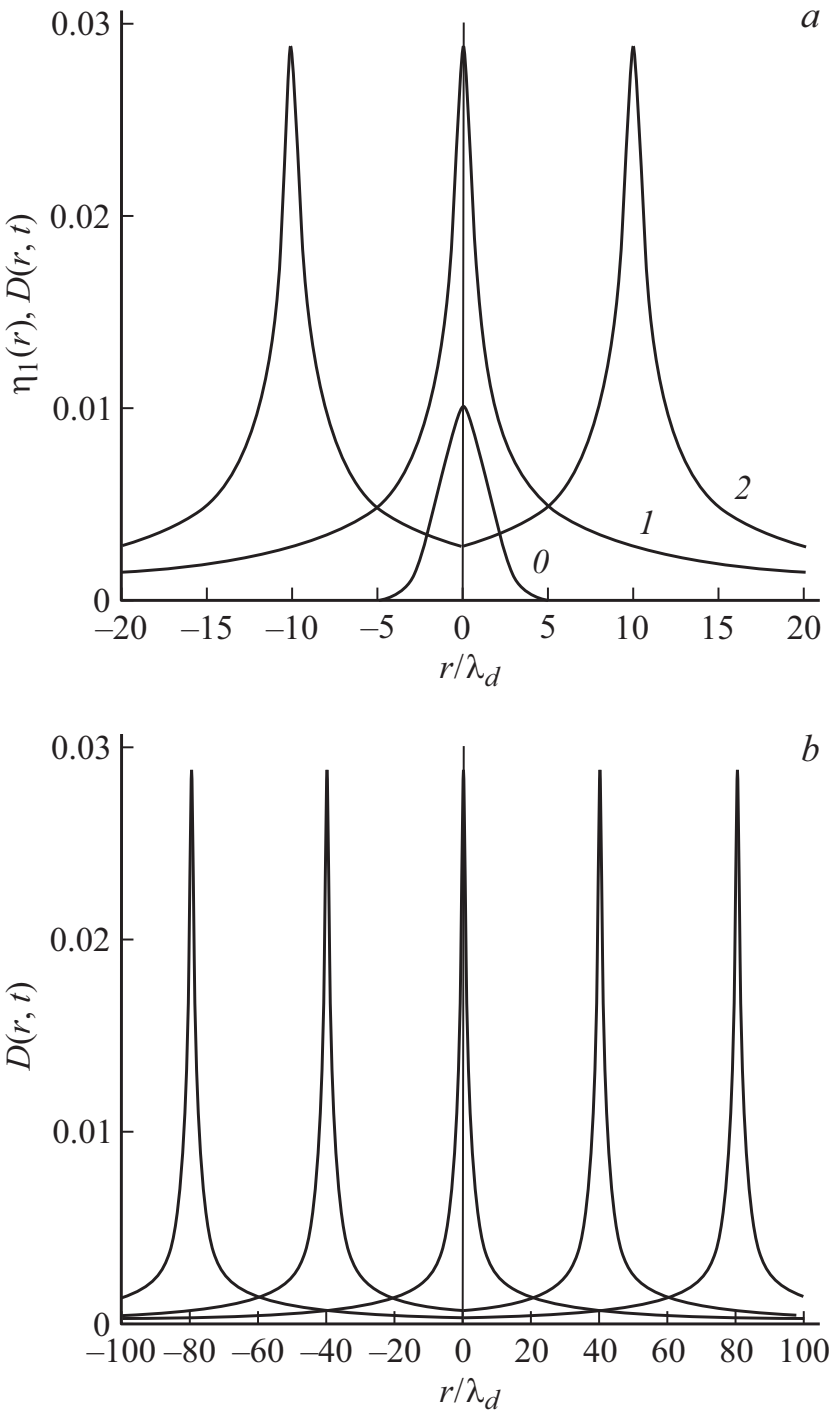

Pис. 5. Дискретные дислокации МП (кривые 1 и 2) вблизи источника наномодуляции кристалла (кривая 0$)(a)$ и вдали от него $(b)$.

их образования,

$$
D(r, t)=2 \pi \int_{-Z_{2}}^{Z_{2}} \frac{\varepsilon\left(z_{2}, z_{0}\right) d z_{2}}{\|\tilde{r}|-| \tilde{t}\|+\tilde{r}_{0}},
$$

где $D(r, t)$ - распределение деформаций в дискретных дислокациях МП, $Z_{2}=\mp 10-$ пределы интегрирования (суммирования) предмартенситных деформаций, $z_{0}=0.5, \xi=6 \cdot 10^{-2}, \tilde{r}=r / \lambda_{d}, \tilde{r}_{0}=r_{0} / \lambda_{d}=1-$ минимальная величина радиуса $r, \tilde{t}=t / \tau$. Зависимость $D(r, t)$ показана на рис. 5, $a$ для двух моментов времени: $t / \tau=0$ (кривая 1 ) и $t / \tau=10$ (кривая 2). Видно, что дискретные петли дислокаций МП появляются вначале вблизи источника наномодуляции кристалла (рис. 2). На рис. 5, а его иллюстрирует кривая, обозначенная символом 0. С течением времени $(t / \tau=20$ и 80$)$ петли все дальше удаляются от своего источника (рис. $5, b)$. Видно также, что максимальные деформации в петлях $\approx 3 \%$ имеют тот же порядок величины, что и в эксперименте.

\section{5. Заключение}

Таким образом, в рамках теории Гинзбурга-Ландау для неравновесных фазовых переходов первого рода и разумных предположений относительно пространственной консолидации локальных упругих полей предмартенситной наноструктуры кристалла продемонстрирован возможный ее переход в структуру дискретных дислокационных петель мартенситного превращения.

\section{Конфликт интересов}

Автор заявляет, что у него нет конфликта интересов.

\section{Список литературы}

[1] Г.В. Курдюмов, Л.Г. Хандрос. ДАН СССР 66, 211 (1949).

[2] B.A. Bilby. Phil. Mag. 44, 782 (1953).

[3] F.C. Frank. Acta Met. 1, 15 (1953).

[4] A. Sato, N. Mori. Mater. Sci. Eng. A 146, 197 (1991).

[5] W.S. Ko, S.B. Maisel, B. Grabovsski, J.B. Jcon. J. Neugebauer. Acta Mater. 123, 90 (2017).

[6] P.C. Hoenberg, A.P. Krekhov. Phys. Rep. 572, 1 (2015).

[7] Г.А. Малыгин. УФН 171, 187 (2001).

[8] Г.А. Малыгин. ФТТ 43, 1911 (2001).

[9] Y. Noda, M. Takimoto, T. Nakagava, Y. Yamada. Met. Trans. A 19, 265 (1988).

Редактор Ю.Э. Китаев 